http://dx.doi.org/10.35381/racji.v5i3.1101

\title{
Ley Orgánica Integral para la Prevención y Erradicación de la Violencia de
} Género contra las Mujeres

\section{Comprehensive Organic Law for the Prevention and Eradication of Gender Violence against Women}

\author{
Marcia Esther España-Herrería \\ ub.marciaespana@uniandes.edu.ec \\ Universidad Regional Autónoma de los Andes, Babahoyo \\ Ecuador \\ https://orcid.org/0000-0001-7818-9577 \\ Ariana Katherine Galarza-Quinto \\ kattiari17@gmail.com \\ Universidad Regional Autónoma de los Andes, Babahoyo \\ Ecuador
}

Recibido: 31 de octubre de 2020

Revisado: 10 de octubre de 2020

Aprobado: 05 de diciembre de 2020

Publicado: 10 de diciembre de 2020 
lustitia Socialis. Revista Arbitrada de Ciencias Jurídicas.

Año V. Vol. V. №3. Edición Especial. 2020-III:

Universidad Regional Autónoma de los Andes

Hecho el depósito de Ley: FA2016000064

ISSN: 2542-3371

FUNDACIÓN KOINONIA (F.K). Santa Ana de Coro, Venezuela

Marcia Esther España-Herrería; Ariana Katherine Galarza-Quinto

\title{
RESUMEN
}

La sociedad ecuatoriana ha sido testigos de la constante agresión que han sufrido las mujeres, muchas veces por su propia pareja sentimental, pese a que el Estado ha creado leyes para sancionarlo, esto no ha sido suficiente para reducir la violencia contra la mujer. La presente investigación tiene como objetivo analizar el cumplimiento de la Ley Orgánica Integral para Prevenir y Erradicar la Violencia Contra la Mujer en caso de hechos de violencia contra la mujer en el Cantón Babahoyo Ecuador. La investigación se realizará desde la perspectiva cuantitativa, con una metodología descriptiva y un diseño no experimental, apoyándose en el análisis documental y bibliográfico Para la recopilación de los datos se realizó mediante una encuesta y se aplicó un cuestionario. Se concluye que poco ha logrado avanzar en el cumplimiento de la norma por lo que se propone la innovación de la ordenanza de prevención, atención, y erradicación de la violencia y discriminación.

Descriptores: Violencia contra la mujer; derecho de la familia; descentralización. (Palabras tomadas del Tesauro UNESCO).

\begin{abstract}
Ecuadorian society has witnessed the constant aggression that women have suffered, many times by their own sentimental partner, despite the fact that the State has created laws to sanction it, this has not been enough to reduce violence against women. The present research aims to analyze compliance with the Comprehensive Organic Law to Prevent and Eradicate Violence Against Women in the event of acts of violence against women in the Canton Babahoyo Ecuador. The research will be carried out from a quantitative perspective, with a descriptive methodology and a non-experimental design, relying on documentary and bibliographic analysis. Data collection was carried out through a survey and a questionnaire was applied. It is concluded that little progress has been made in complying with the standard, which is why it is proposed to innovate the ordinance of prevention, care, and eradication of violence and discrimination.
\end{abstract}

Descriptors: Violence againt women; family law; decentralization. (Words taken from the UNESCO Thesaurus). 
lustitia Socialis. Revista Arbitrada de Ciencias Jurídicas.

Año V. Vol. V. №3. Edición Especial. 2020-III:

Universidad Regional Autónoma de los Andes

Hecho el depósito de Ley: FA2016000064

ISSN: 2542-3371

FUNDACIÓN KOINONIA (F.K). Santa Ana de Coro, Venezuela

Marcia Esther España-Herrería; Ariana Katherine Galarza-Quinto

\section{INTRODUCCIÓN}

La implementación de la Ley Orgánica Integral para Prevenir y Erradicar la Violencia contra las Mujeres (2018), claramente es un aporte vital, sin embargo, es importante identificar qué herramientas ha tomado el Gobierno Autónomo Descentralizado de Babahoyo para su aplicación y efectividad. En virtud de esto, se analizará si existe la necesidad de crear nuevas políticas públicas que aporten a esta Ley, ya que la simple creación de normas sancionadoras no previene ni erradica el problema. Sin embargo, es necesario investigar las coyunturas administrativas y jurídicas que podrían ser perfectibles, en aras de disminuir y excluir de manera permanente la vulneración de los derechos de las mujeres de este cantón. Para Berni (2018) La violencia de género es todo acto de violencia sexista que tiene como resultado posible o real un daño físico, psicológico, sexual. Con todo el peso de la ley, se debe luchar para evitar la violencia de género.

La importancia de ponderar el estado actual de la aplicación de la ley permite reconocer, justo antes del cambio de las autoridades seccionales; los avances y logros obtenidos en la administración saliente y, a su vez, verificar el cumplimiento de una norma jurídica. La Constitución Nacional (2018), claramente su artículo 70 tipifica:

El Estado formulará y ejecutará políticas para alcanzar la igualdad entre mujeres y hombres, a través del mecanismo especializado de acuerdo con la ley, e incorporará el enfoque de género en planes y programas, y brindará asistencia técnica para su obligatoria aplicación en el sector público.

Así mismo, una de las tareas principales del Derecho Procesal Penal, es la prevención de la violencia hacia la mujer por parte de su pareja, o cualquier miembro de su núcleo familiar. En el Código Orgánico de la Función Judicial y el Código Orgánico Integral Penal, se establecen los procedimientos para sustanciar este tipo de causas, respetando el cumplimiento integral del debido proceso. Si bien el paso de tiempo ha sido significativo en favor a la mujer con Leyes que garantizan y protegen sus derechos, no es menos cierto que la violencia aún se evidencia dentro de los distintos estratos sociales y que esta lucha seguirá en pie hasta que las Leyes sean acatadas en su totalidad y donde además se apliquen mecanismos preventivos eficaces. 
Los derechos de las víctimas de violencia intrafamiliar han tenido durante los últimos años un avance importante que se ha reflejado con la constante modificación de las leyes con la finalidad de mejorar la protección que el estado da a este grupo vulnerable. Sin embargo, desde tiempos muy remotos ha existido la violencia contra la mujer y la familia en general, la mujer ha tenido que tolerar el agravio incesante de sus parejas, ya sea por salvaguardar a sus hijos o por sentirse desamparada económicamente. El maltrato no solamente ha sido físico sino psicológico el cual afecta a su autoestima en primer lugar, lo que la hace un blanco fácil para la violencia (Castillo-Saavedra, Bernardo-Trujillo, \& Medina-Reyes, 2018).

Según datos del Instituto Nacional de Estadísticas y Censos (INEC, 2017) desde la década de los 80 grupos de mujeres han protestado al respecto y es recién en los 90 que se dieron cambios importantes, como la creación de las Comisarías de la Mujer y la Familia; en el año 1995 se estableció la nueva la Ley contra la violencia a la mujer y la familia; sin embargo, las cifras no son alentadoras, pues se reconoce que al menos 6 de cada 10 mujeres han sido víctimas de violencia, siendo la psicológica la mayor en el $53,9 \%$.

Podemos observar que, a pesar de la existencia del Código Orgánico Integral Penal, no se han logrado reducir notablemente los índices de violencia hacia la mujer; ante esta realidad, la Asamblea Nacional del Ecuador en el año 2018 aprobó la Ley Orgánica Integral para Prevenir y Erradicar la Violencia Contra las Mujeres, una normativa que cumple un rol muy amplio en lo que involucra la defensa de la víctima, en la que interviene el Estado y otorga corresponsabilidad a la sociedad, para afrontar este problema en tres grandes campos: la atención inmediata, la protección de la víctima y la reparación para que pueda restablecerse de mejor manera a una vida plena.

En tal sentido prescribe en la Constitución de la Republica (2018) en su Artículo 5.Obligaciones estatales. El Estado, a través de todos los niveles de gobierno, tiene las obligaciones ineludibles de promover, proteger, garantizar y respetar los derechos humanos de las mujeres: niñas, adolescentes, adultas y adultas mayores, a través de la adopción de todas las medidas políticas, legislativas, judiciales, administrativas, de 
control y de cualquier otra índole que sean necesarias, oportunas y adecuadas para asegurar el cumplimiento de la presente Ley y se evite la revictimización e impunidad. Estas obligaciones estatales constarán en el Plan Nacional de Desarrollo y en los Planes de Desarrollo: regionales, provinciales, de los distritos metropolitanos, cantonales y parroquiales; $y$, se garantizarán a través de un plan de acción específico incluido en el Presupuesto General del Estado.

Por ello, desde el 5 de febrero de 2018, fecha en que se promulgó la ley, el Estado asumió una serie de acciones a ejecutar que conllevan la creación de una serie de estructuras administrativas, técnicas y especializadas, materializadas inicialmente por un Sistema nacional que prevenga y erradique la violencia contra las mujeres como ente rector, que vincule a entidades públicas que ofrecerían un paraguas de protección a las mujeres víctimas de violencia; entre las estructuras propuestas se encuentran: el Registro Único de Violencia, el Observatorio Nacional; en total 15 organizaciones regidas por el gobierno central en sus diferentes funciones y un delegado de los Gobiernos Autónomos Descentralizados.

La presente investigación tiene como objetivo analizar el cumplimiento de la ley en caso de hechos de violencia contra la mujer en el Cantón Babahoyo Ecuador.

\section{METODOLOGÍA}

La investigación se realizará desde la perspectiva cuantitativa, con una metodología descriptiva y un diseño no experimental, apoyándose en el análisis documental y bibliográfico lo que permitió examinar documentos, normas, leyes, investigaciones relacionados con el tema. Permitiendo organizar mediante el método analíticosintético, el argumento teórico jurídico relacionado al tema. Para la recopilación de los datos se realizó mediante una encuesta y se aplicó un cuestionario. La población con la que se trabajó esta investigación fueron los empleados de la Junta Cantonal de Protección de Derechos del Gobierno Autónomo Descentralizado del cantón Babahoyo, Defensor Público, Jueces de la Unidad Judicial de la Familia, mujer, niñez y adolescencia y jueces de Violencia a la mujer y miembros del núcleo familiar de esta ciudad de Babahoyo. El universo de la población considerado en el cantón Babahoyo 
es de 14 personas, por lo cual la muestra será de 14 personas. Distribuidos de la siguiente manera: Junta Cantonal de Protección de Derecho (4), Jueces de la Unidad Judicial de la Familia, Mujer, Niñez y Adolescencia (6), Defensor Público (2) y Jueces de Violencia contra la Mujer y miembros del núcleo familiar (2).

\section{RESULTADOS}

A continuación, se presentan los resultados obtenidos:

\section{Cuadro 1.}

Estadísticas de delitos contra la mujer en Babahoyo 2017-2018.

\begin{tabular}{lc}
\hline DELITOS CONTRA LA MUJER & 04/06/2017- 03/06/2018 \\
\hline ABUSO SEXUAL & 18 \\
VIOLACIÓN & 29 \\
FEMICIDIO & 4 \\
PROSTITUCIÓN FORZADA & 1 \\
VIOLENCIA FÍSICA & 88 \\
VIOLENCIA PSICOLÓGICA & 18 \\
TOTAL & $\mathbf{1 5 8}$ \\
\hline
\end{tabular}

Fuente: Estadísticas del Consejo de la Judicatura (2019).

De acuerdo a los datos que se observan en la tabla №1 en el período 2017 al 2018 se registraron hechos de violencia contra la mujer en el Cantón Babahoyo, resaltando la violencia física, seguido de los hechos de violación y abuso sexual, la violencia psicológica, femicidio y prostitución forzada, para un total 158 casos, lo que demuestra que el cumplimiento de la norma debe exigirse con mayor ahínco en cumplimiento y observancia de la ley. 
lustitia Socialis. Revista Arbitrada de Ciencias Jurídicas.

Año V. Vol. V. №3. Edición Especial. 2020-III:

Universidad Regional Autónoma de los Andes

Hecho el depósito de Ley: FA2016000064

ISSN: 2542-3371

FUNDACIÓN KOINONIA (F.K). Santa Ana de Coro, Venezuela

Marcia Esther España-Herrería; Ariana Katherine Galarza-Quinto

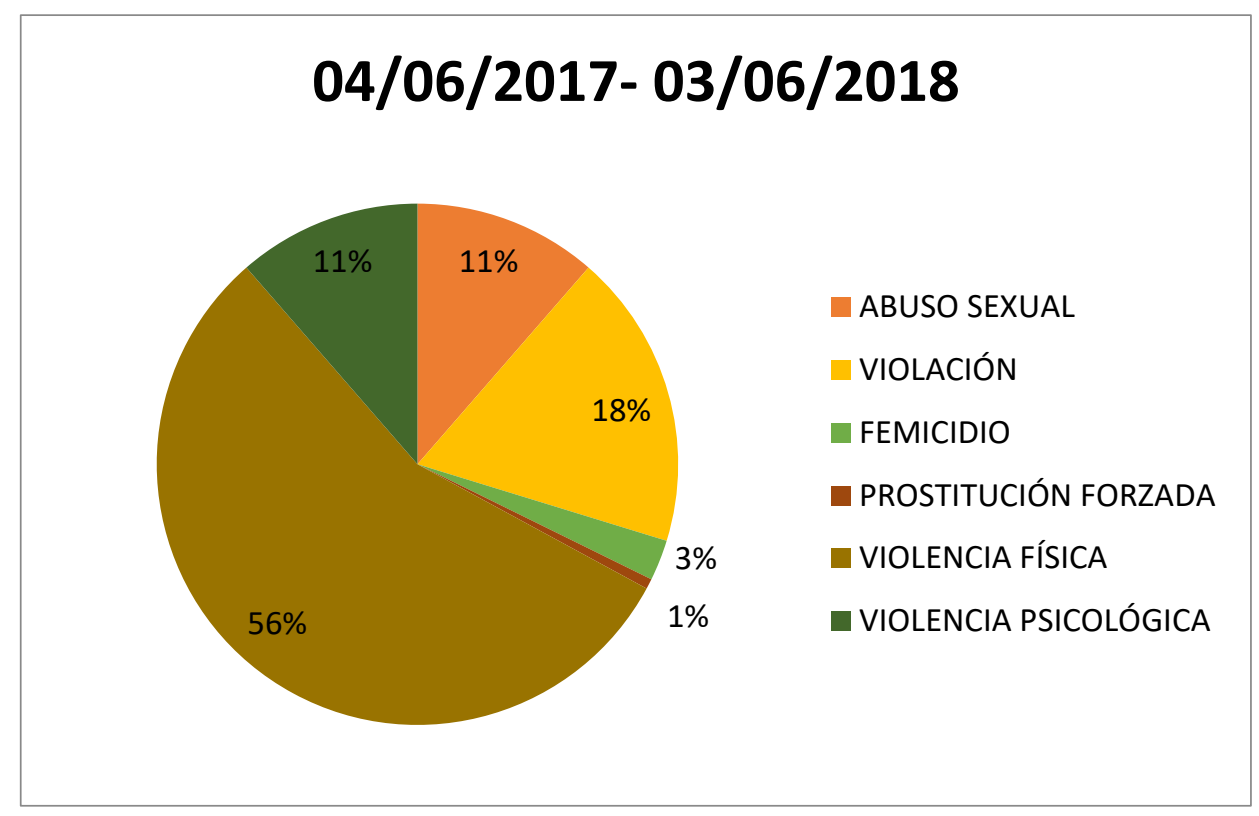

Gráfico 1. Índice de violencia Babahoyo 2017-2018.

Fuente: Elaboración propia (2020).

9

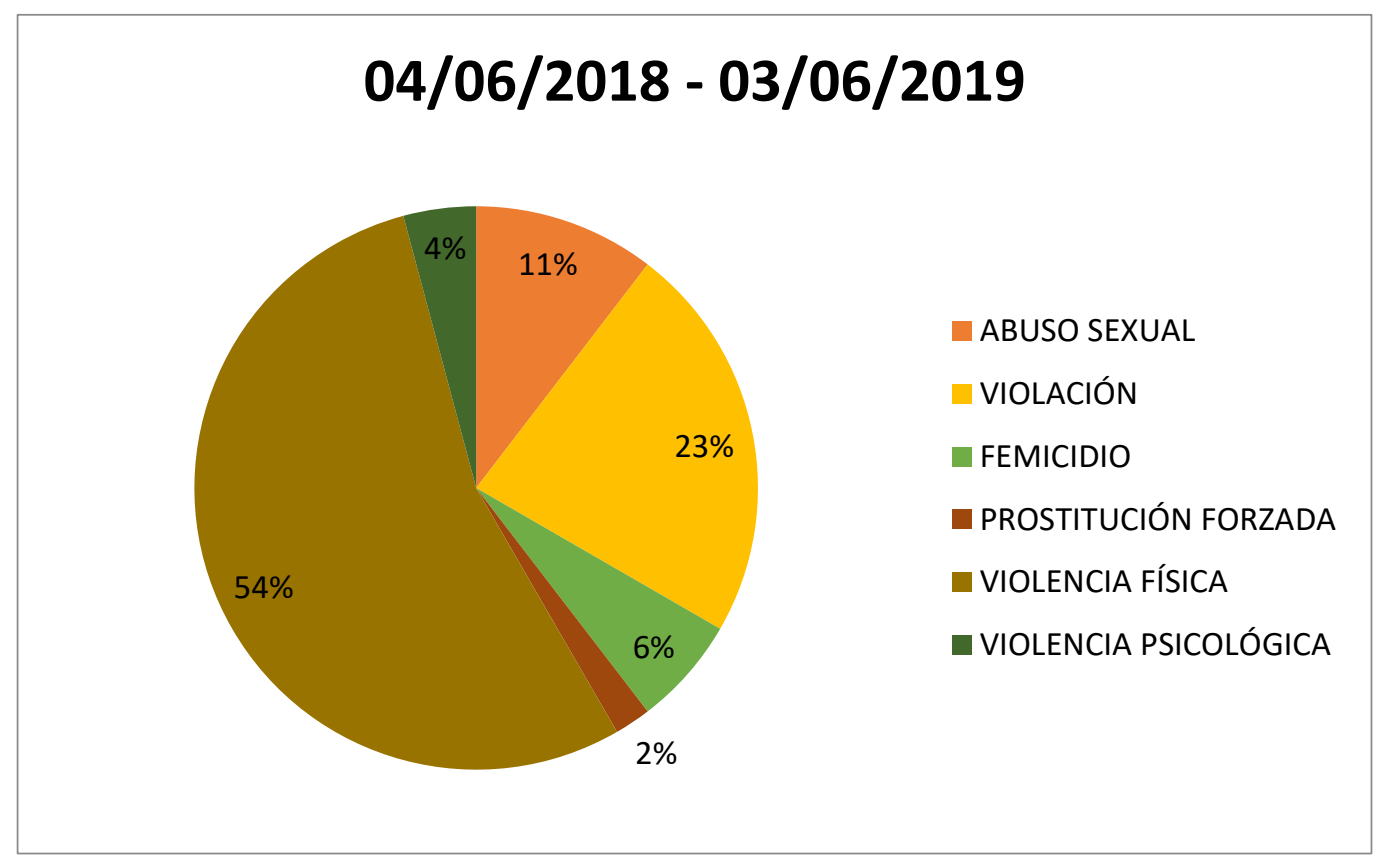

Gráfico 2. Índices de violencia Babahoyo 2018-2019.

Fuente: Elaboración propia (2020). 
Al observar las gráficas 1 y 2 el alto porcentaje corresponde a la violencia física en un $56 \%$ y $54 \%$ respectivamente, agrupando lo relacionado a lo sexual alcanza un $66 \%$, los femicidios $9 \%$ y un $15 \%$ violación psicológica. Es alarmante lo representado en las gráficas ya que indica una deficiencia en las políticas que se están desarrollando este esta comunidad y como se violentan la ley. Para Carrillo-Kennedy (2018) La problemática del femicidio está cobrando cada día mayor importancia en la sociedad ecuatoriana y latinoamericana, debido al crecimiento de estos crímenes a nivel nacional, cuyo fenómeno afecta directamente al desarrollo de la población femenina y se opone a los principios constitucionales, por lo que es necesario que el Estado a través del cumplimiento de la legislación en materia penal impida la impunidad de los criminales y controle adecuadamente el orden social para ofrecer calidad de vida digna a las mujeres del Ecuador. (p.132).

\section{DISCUSIÓN}

Entre las atribuciones que tienen todos los gobiernos autónomos descentralizados con respecto a la Ley Orgánica para prevenir y erradicar la violencia de género, establecidas en su artículo 38, encontramos las de ejecutar, diseñar y formular normativas y políticas públicas locales para la prevención y erradicación de la violencia de genero contra las mujeres, también formular y ejecutar resoluciones, planes y programas para la prevención y erradicación de la violencia contra las mujeres. Estas atribuciones si han sido ejecutadas dentro del gobierno autónomo descentralizado del cantón Babahoyo. En tal sentido se debe realizar un esfuerzo por disminuir la violencia como lo afirman Aldana-Zavala \& Valles-González (2018:111) Contrarrestar la violencia en contra de la mujer, implica trabajar día a día en fomentar una cultura de prevención en favor del género y de su dignidad como persona.

Por otro lado, entre otras de las atribuciones que tienen los GAD'S tenemos las de crear y fortalecer las juntas cantonales de protección de derechos, es decir capacitar al personal en atención y emisión de medidas. Promover la creación de Centros de Equidad y Justicia para la protección de derechos y brindar atención a las mujeres víctimas de violencia de género, con equipos técnicos especiales; en cuanto a estas 
atribuciones, podemos observar que no están cumpliéndose dentro de nuestro gobierno autónomo descentralizado.

Otras atribuciones que tienen los GAD'S que no se cumplen es establecer mecanismos para la detección y derivación a las instituciones del sistema, dirigidas a la comunidad; diseñar e implementar un sistema de recolección de información sobre casos de violencia de genero contra las mujeres, evaluar de manera periódica a nivel de satisfacción de las usuarias en los servicios de atención especializadas para las víctimas y remitir información necesaria para la construcción de estadísticas referentes a tipo de infracción.

Es importante señalar, que la ley establece que los gobiernos autónomos descentralizados deben desarrollar mecanismos comunitarios o barriales de prevención como alarmas, rondas de vigilancia y acompañamiento a las mujeres víctima de violencia, en conjunto a la policía nacional.

Sin embargo, los GAD'S deben de promover iniciativas locales como mesa intersectorial de violencia, sistema provincial integrado de prevención y atención de las víctimas de violencia de género como los servicios de atención en casos de violencia de género. Y la última atribución de tal institución es definir los instrumentos para el estricto control de todo espectáculo público a fin de que se prohíba, suspenda o clausure aquellos que estén promoviendo la violencia o discriminación hacia las mujeres, en todas estas atribuciones podemos observar que la ley no está siendo aplicada en el Gobierno Autónomo Descentralizado del Cantón Babahoyo.

\section{CONCLUSIONES}

El avance en el reconocimiento de los derechos humanos ha logrado que, en el último siglo, se reconozca de manera clara la problemática de la degradación y violencia dada a la mujer a lo largo de la historia, esto ha repercutido en la creación de normas especiales para proteger la libertad de las mujeres a través de tratados y convenciones mundiales que en contexto son la base para las legislaciones de las naciones suscritas a estos acuerdos. 
Con el desarrollo de estas nuevas perspectivas jurídicas, la legislación ecuatoriana ha ido acogiendo estos cambios, que conllevan principalmente la erradicación, prevención y atención a las mujeres víctimas de violencia en cualquiera de sus presentaciones, logrando perfeccionarse en una norma específica de ley que aglutina los procedimientos antes, durante y después de un incidente vulnerador de derechos. Sin embargo, poco más de un año después de la emisión de la ley de prevención y erradicación de la violencia contra la mujer, el Gobierno Autónomo Descentralizado de Babahoyo, con las Juntas Cantonales de Protección de Derecho. Poco ha logrado avanzar en el cumplimiento de la norma por lo que se propone la innovación de la ordenanza de prevención, atención, y erradicación de la violencia y discriminación basada en género del cantón Babahoyo, que brinde agilidad al desarrollo de las obligaciones dispuestas en la ley para proteger el derecho violado o restituir el derecho a la mujer.

\section{FINANCIAMIENTO}

No monetario.

\section{AGRADECIMIENTO}

A la Universidad Regional Autónoma de Los Andes; por motivar el desarrollo de la Investigación.

\section{REFERENCIAS CONSULTADAS}

Aldana-Zavala, J. \& Valles-González, E. (2018) La mujer en Derecho a una Vida libre de violencia desde la Organización Comunitaria. [Women in the Right to a Life Free of Violence from the Community Organization]. IUSTITIA SOCIALIS. 3(5),110-127. doi:http://dx.doi.org/10.35381/racii.v3i5.171

Asamblea Nacional de la República del Ecuador. (2014). Código Orgánico Integral Penal. [Comprehensive Organic Criminal Code]. Recuperado de https://n9.cl/g6sc 
lustitia Socialis. Revista Arbitrada de Ciencias Jurídicas.

Año V. Vol. V. №3. Edición Especial. 2020-III:

Universidad Regional Autónoma de los Andes

Hecho el depósito de Ley: FA2016000064

ISSN: 2542-3371

FUNDACIÓN KOINONIA (F.K). Santa Ana de Coro, Venezuela

Marcia Esther España-Herrería; Ariana Katherine Galarza-Quinto

Asamblea Nacional del Ecuador. (05 de febrero de 2018). Ley Orgánica Integral para prevenir y erradicar la violencia contra las mujeres. Tipos de Violencia. [Comprehensive Organic Law to prevent and eradicate violence against women. Types of Violence]. Quito, Pichincha, Ecuador: Registro Oficial Suplemento 175 de 05-feb.-2018.

Asamblea Nacional Constituyente de la República del Ecuador, (2008). Constitución de la República del Ecuador. [Constitution of the Republic of Ecuador]. Montecristi. Registro Oficial 449 de 20-oct-2008. Recuperado de https://n9.cl/sia

Asamblea Nacional (2010). Código Orgánico de Organización Territorial, Autonomía y Descentralización. [Organic Code of Territorial Organization, Autonomy and Decentralization]. Registro oficial 303 de 19 de oct-2010

Carrillo-Kennedy, J. (2018). Incidencia de femicidio en el Ecuador y en la provincia del Guayas. [Incidence of femicide in Ecuador and Guayas province]. Revista Universidad y Sociedad, 10(1), 125-133.

Castillo-Saavedra, E, Bernardo-Trujillo, J, \& Medina-Reyes, M. (2018). Violencia de género y autoestima de mujeres del centro poblado Huanja - Huaraz, 2017 [Gender violence and self-esteem in women from the Huanja - Huaraz hamlet, 2017]. Horizonte Médico (Lima), 18(2), 47-52. https://dx.doi.org/10.24265/horizmed.2018.v18n2.08

COFJ. (2009). Código Orgánico de la Función Judicial. [Organic Code of Judicial Function] Quito: Registro Oficial suplemento 544. 09-Mar-2009.

Instituto Nacional de Estadísticas y Censos. (2017). Violencia de Género. [Gender Violence]. Recuperado de www.inec.gob.ec

Patricia-Berni, M. (2018). Estrativismo social machista en Ecuador violencia de género, femicidio. [Machist social estrativism in Ecuador gender violence, femicide] Conrado, 14(61),111-115.

(C2020 por los autores. Este artículo es de acceso abierto y distribuido según los términos y condiciones de la licencia Creative Commons Atribución-NoComercial-Compartirlgual 4.0 Internacional (CC BY-NC-SA 4.0)

(https://creativecommons.org/licenses/by-nc-sa/4.0/). 